\title{
Explanation for papers missing from this special issue
}

\author{
Atilla Ansal ${ }^{1}$
}

(C) Springer Science+Business Media B.V., part of Springer Nature 2018

As a result of an editorial mistake five papers intended for this special issue ended up in Bulletin of Earthquake Engineering 15(11), 2017. For completeness we hereby provide their publication information as well as their abstracts. Our sincere apologies for this.

1. Toward an empirical ground motion prediction equation for France: accounting for regional differences in the source stress parameter
Gabriele Ameri ${ }^{1 \text { t. }}$
Stéphane Drouet ${ }^{1}$
Paola Traversa ${ }^{2}$
Dino Bindi ${ }^{3}$
Fabrice Cotton ${ }^{3}$

Ameri, G., Drouet, S., Traversa, P. et al. Bull Earthquake Eng (2017) 15: 4681. https://doi. org/10.1007/s10518-017-0171-1

g.ameri@fugro.com

1. Geoter SAS - Fugro, Auriol, France

2. EDF, CEIDRE/TEGG/SGG, Aix-en-Provence, France

3. German Research Centre for Geosciences, GFZ, Potsdam, Germany

\begin{abstract}
Atilla Ansal

atilla.ansal@ozyegin.edu.tr

1 School of Engineering, Ozyegin University, Istanbul, Turkey
\end{abstract}

In low-to-moderate seismicity regions such as metropolitan France, characterized by limited strong-motion records in the magnitude-distance range of interest for seismic hazard 
assessment, the derivation of empirical ground motion prediction equations (GMPEs) is a major challenge. In this study, we take advantage of the RESORCE-2013 database (http://resorce-portal.eu/) that contains uniformly processed records for the Pan-European region including relevant number of French records. After discussing the metadata for French events and stations, we first derive a base-case GMPE that is used to investigate the within-event and between-event residuals. The short-period between-event residuals for French (and Swiss) events show larger variability with respect to larger magnitude events in other regions. We show that the between-event residuals are clearly correlated with the stress parameter and that such larger variability can be explained by accounting for stress-parameter scaling. We derive an empirical scaling of ground motion with stress parameter that is consistent across regions and with the scaling predicted by stochastic GMPEs. This suggests that the scaling of ground motion with stress parameter for a given magnitude is largely region independent whereas the absolute stress parameter values may vary regionally. Based on these results we propose to adopt the scaling model as a function of stress parameter and magnitude by Yenier and Atkinson (Bull Seismol Soc Am 105(4):1989-2009, 2015) by adapting the reference stress parameter to our target regions. By accounting for stress parameter scaling in the GMPE we reduce the between-event variability for French and Swiss small-magnitude events. Finally, we investigate the aleatory variability $(\sigma)$ of the GMPE and its components $\left(\tau, \phi, \phi_{\mathrm{ss}}\right)$. We propose a heteroscedastic $\sigma$ model to be used when the stress-parameter scaling is not considered in the GMPEs due to lack of information. If enough information on the stress-parameter is available the adjusted GMPE can be applied using a homoscedastic $\sigma$. Despite using small events, the $\phi_{\mathrm{ss}}$ for French stations is found to be consistent with other studies and confirms the stability of $\phi_{\mathrm{ss}}$ across different regions and datasets.

\title{
2. Stochastic source, path and site attenuation parameters and associated variabilities for shallow crustal European earthquakes
}

\author{
Sanjay Singh Bora ${ }^{1 \text { ब }}$ \\ Fabrice Cotton ${ }^{1,2}$ \\ Frank Scherbaum ${ }^{1,2}$ \\ Benjamin Edwards ${ }^{3}$ \\ Paola Traversa ${ }^{4}$
}

Bora, S.S., Cotton, F., Scherbaum, F. et al. Bull Earthquake Eng (2017) 15: 4531. https:// doi.org/10.1007/s10518-017-0167-x

bora@gfz-potsdam.de

1. GFZ German Research Center for Geosciences, Potsdam, Germany

2. University of Potsdam, Potsdam, Germany

3. Department Earth, Ocean and Ecological Sciences, University of Liverpool, Liverpool, UK

4. French Electric Company, Aix-en-Provence, France

\section{Abstract}

We have analyzed the recently developed pan-European strong motion database, RESORCE-2012: spectral parameters, such as stress drop (stress parameter, $\Delta \sigma$ ), anelastic 
attenuation $(Q)$, near surface attenuation $\left(\kappa_{0}\right)$ and site amplification have been estimated from observed strong motion recordings. The selected dataset exhibits a bilinear distancedependent $Q$ model with average $\kappa_{0}$ value 0.0308 s. Strong regional variations in inelastic attenuation were also observed: frequency-independent $Q_{0}$ of 1462 and 601 were estimated for Turkish and Italian data respectively. Due to the strong coupling between $Q$ and $\kappa_{0}$, the regional variations in $Q$ have strong impact on the estimation of near surface attenuation $\kappa_{0}$. $\kappa_{0}$ was estimated as 0.0457 and $0.0261 \mathrm{~s}$ for Turkey and Italy respectively. Furthermore, a detailed analysis of the variability in estimated $\kappa_{0}$ revealed significant within-station variability. The linear site amplification factors were constrained from residual analysis at each station and site-class type. Using the regional $Q_{0}$ model and a site-class specific $\kappa_{0}$, seismic moments $\left(M_{0}\right)$ and source corner frequencies $f_{c}$ were estimated from the site corrected empirical Fourier spectra. $\Delta \sigma$ did not exhibit magnitude dependence. The median $\Delta \sigma$ value was obtained as 5.75 and $5.65 \mathrm{MPa}$ from inverted and database magnitudes respectively. A comparison of response spectra from the stochastic model (derived herein) with that from (regional) ground motion prediction equations (GMPEs) suggests that the presented seismological parameters can be used to represent the corresponding seismological attributes of the regional GMPEs in a host-to-target adjustment framework. The analysis presented herein can be considered as an update of that undertaken for the previous Euro-Mediterranean strong motion database presented by Edwards and Fäh (Geophys J Int 194(2):1190-1202, 2013a).

3. Systematic source, path and site effects on ground motion variability: the case study of Northern Italy

\author{
Giovanni Lanzano ${ }^{1 \bowtie}$ \\ Francesca Pacor ${ }^{1}$ \\ Lucia Luzi ${ }^{1}$ \\ Maria D'Amico ${ }^{1}$ \\ Rodolfo Puglia ${ }^{1}$ \\ Chiara Felicetta ${ }^{1}$
}

Lanzano, G., Pacor, F., Luzi, L. et al. Bull Earthquake Eng (2017) 15: 4563. https://doi. org/10.1007/s10518-017-0170-2

giovanni.lanzano@ingv.it

1. Istituto Nazionale di Geofisica e Vulcanologia, Milan, Italy

\begin{abstract}
In this paper, we separate the residuals of ground motion prediction equations into different components, calculating the repeatable site-specific $\left(\delta \mathrm{S} 2 \mathrm{~S}_{\mathrm{s}}\right)$, location-specific $\left(\delta \mathrm{L}_{2} \mathrm{~L}_{\mathrm{r}}\right)$ and path-specific $\left(\delta \mathrm{P} 2 \mathrm{P}_{\mathrm{er}}\right)$ correction for the median model. In this way, we can reduce the total standard deviation (sigma) of the ground-motion model by Lanzano et al. (Bull Seismol Soc Am 106(1):73-92, 2016), removing these components and overcome the ergodic assumption. These repeatable terms are empirically estimated using a dataset composed by acceleration waveforms of Italian events mainly occurred in the Po plain and Eastern Alps (Northern Italy), in the time interval 1976-2015. The analysis is carried out on 2241 recordings, from 88 shallow earthquakes recorded by 168 sites. A local model, specifically
\end{abstract}


tailored for Northern Italy, is used as the reference GMPE, which predicts the geometric mean of the horizontal peak ground accelerations and response spectral ordinates in the period range $0.04-4 \mathrm{~s}$. Because most of the propagation paths sampled only once, the path-specific components and the resulting aleatory variability are also calculated from the spatial correlation of residuals, following the approach of Lin et al. (Bull Seismol Soc Am 101(5):2281-2295, 2011). We find a significant reduction of the overall ground motion variability to an extent comparable to what observed from previous studies on empirical and simulated datasets. The estimated median corrections and variance components can be used in probabilistic seismic hazard assessment for a single site and a single path in Northern Italy. As an example, we compute the hazard curves at some sites by means of three different approaches (ergodic, partially non-ergodic and full non-ergodic), in order to quantify the impact of relaxing the ergodic assumption.

\title{
4. Comparison of PSH results with historical macroseismic observations at different scales. Part 1: methodology
}

M. $\operatorname{Rota}^{1 \times}$

A. Rosti ${ }^{2}$

Rota, M. \& Rosti, A. Bull Earthquake Eng (2017) 15: 4585. https://doi.org/10.1007/s1051 8-017-0157-z

maria.rota@eucentre.it

1. European Centre for Training and Research in Earthquake Engineering, Pavia, Italy

2. ROSE Programme, UME School, IUSS Pavia, Pavia, Italy

\begin{abstract}
The need for verifying and/or validating the results of probabilistic seismic hazard studies has become evident, despite the absence of generally accepted methods for judging such results. This paper proposes a methodology for comparing the results of PSH with historical macroseismic observations, at different scale levels. The selected metric of the comparison is the mean damage, i.e. the average annually expected damage. Hence, a procedure for converting macroseismic observations and PGA levels, for which PSH estimates are provided, into mean damage values is first presented. The procedure is based on the macroseismic method for converting intensities into mean damage values, whereas it takes advantage of fragility curves (representative of the seismic vulnerability of the building stock at the time of historical observations) to transform the rates of occurrence of PGA values into the equivalent quantity in terms of mean damage. A methodology for site-specific comparison is first outlined. To overcome some of the limitations of the comparison at a single site, two procedures for aggregating several sites by sampling in space are then presented, with the aim of enlarging the available macroseismic dataset. Finally, a procedure for the comparison at the regional level is discussed. Applications of the proposed methodologies will be discussed in a companion paper (Rosti and Rota in Bull Earthq Eng, 2017).
\end{abstract}


5. Comparison of PSH results with historical macroseismic observations at different scales. Part 2: application to South-East France
A. Rosti ${ }^{1}$
M. $\operatorname{Rota}^{2 \times}$

Rosti, A. \& Rota, M. Bull Earthquake Eng (2017) 15: 4609. https://doi.org/10.1007/s1051 8-017-0161-3

maria.rota@eucentre.it

1. ROSE Programme, UME School, IUSS Pavia, Pavia, Italy

2. European Centre for Training and Research in Earthquake Engineering, Pavia, Italy

\begin{abstract}
The companion paper by Rota and Rosti (Bull Earthq Eng, 2017) illustrates a methodology for comparing PSH results with historical macroseismic observations at different scales, in terms of mean damage. This paper presents examples of application of the methodology, at the different considered scales, to the South-East quarter of France. This moderate seismicity region is characterised by a long history of civilization, which makes macroseismic observations available for a long time span, although they are highly approximated measures of the seismic action and they are affected by significant uncertainties. The first scale of application presented is at a single site, i.e. the city of Annecy. This example shows that, despite the seismic history of this city is characterised by a significant number of macroseismic observations spanning over a long time period, they mostly consist of very low intensity values and hence the comparison at a single site is not very meaningful. Therefore, the comparison is carried out on a set of seven aggregated sites, well distributed in the region of interest, providing interesting results and suggesting the opportunity of extending the comparison at an even larger scale. The comparison at the regional scale also allows some interesting observations, although it is obviously able to only provide general (average) indications on the PSH results. These comparisons were aimed at showing the applicability of the proposed methodology, pointing out advantages and drawbacks of the different application scales.
\end{abstract}

